EPJ Web of Conferences 107, 02003 (2016)

DOI: $10.1051 /$ epjconf/201610702003

(C) Owned by the authors, published by EDP Sciences, 2016

\title{
Fayans functional for deformed nuclei. Uranium region
}

\author{
S. V. Tolokonnikov ${ }^{1,2, a}$, I. N. Borzov ${ }^{1,3}$, M. Kortelainen ${ }^{4,5}$, Yu. S. Lutostansky ${ }^{1}$, and E. E. Saperstein ${ }^{1,6, b}$ \\ ${ }^{1}$ National Research Centre "Kurchatov Institute", 123182, Moscow, Russia \\ ${ }^{2}$ Moscow Institute of Physics and Technology, 141700, Dolgoprudny, Moscow Region, Russia \\ ${ }^{3}$ Joint Institute for Nuclear Research, 141980 Dubna, Russia \\ ${ }^{4}$ Department of Physics, P.O. Box 35 (YFL), University of Jyvaskyla, Fl-40014 Jyvaskyla, Finland \\ ${ }^{5} \mathrm{Helsinki}$ Institute of Physics, P.O. Box 64, Fl-00014 University of Helsinki, Finland \\ ${ }^{6}$ National Research Nuclear University MEPhl, 115409 Moscow, Russia
}

\begin{abstract}
Fayans energy density functional (EDF) $\mathrm{FaNDF}^{0}$ has been applied to the nuclei around uranium region. Ground state characteristics of the $\mathrm{Th}, \mathrm{U}$ and $\mathrm{Pu}$ isotopic chains, up to the two-neutron drip line, are found and compared with predictions from several Skyrme EDFs. The two-neutron drip line is found for $\mathrm{FaNDF}^{0}$, SLy4 and $\mathrm{SkM}^{*}$ EDFs for a set of elements with even proton number, from $\mathrm{Pb}$ up to $\mathrm{Fm}$.
\end{abstract}

\section{Introduction}

Presently, the Hartree-Fock (HF) or Hartree-Fock-Bogoliubov (HFB) methods together with the effective Skyrme forces [1], Gogny forces [2] or relativistic meanfield (RMF) models [3] are most common microscopical models applied for description of the ground state properties of the heavy nuclei. All of the aforementioned approaches are usually interpreted as a version of the energy density functional (EDF) method suggested by Kohn and Sham [4]. This method is based on the theorem of Hohenberg-Kohn [5], which states that the ground state energy $E_{0}$ of any quantum system is a functional of its density $\rho(\mathbf{r})$. By itself, the theorem says nothing about the form of this functional, and various options for the Skyrme EDF and the Gogny EDF are, in fact, different "ansatzs". Among popular Skyrme EDFs, there are quite old functionals SkM* [6] and SLy4 [7], see the review article [8]. A record in the accuracy of the description of the nuclear masses belongs to the Skyrme functional HFB-17 [9] with the average deviation from the experiment being around $600 \mathrm{keV}$, obtained by adding phenomenological corrections atop of the mean-field. Of a comparable accuracy are the other functionals of this family, up to the HFB-27, the corresponding tables are presented on the site [10]. Also relatively new functional UNEDF1 [11] has proved to be very successful in describing the deformed nuclei.

It is also worth to mention a relatively newly developed approach, known originally as a BCP (Barcelona-CataniaParis) [12] method, and later as a BCPM (BarcelonaCatania-Paris-Madrid) method [13]. The main bulk term of the BCPM functional was found by starting from the equation of the state of nuclear and neutron matter, ob-

\footnotetext{
ae-mail: tolkn@mail.ru

be-mail: saper43_7@mail.ru
}

tained within the Brueckner-Hartree-Fock method by using a realistic $N N+N N N$ potential.

We use the EDF developed by S. A. Fayans with coauthors [14-17]. In comparison to the Skyrme or Gogny EDFs, it possess two main peculiarities. Firstly, the main in-volume term of the Fayans EDF has more sophisticated form. It can be schematically written as

$$
\mathcal{E}(\rho)=\frac{a \rho^{2}}{2} \frac{1+\alpha \rho^{\sigma}}{1+\gamma \rho^{\sigma}}
$$

The corresponding term of the Skyrme EDF would correspond to $\gamma=0$ case within this relation. The use of the bare mass, $m^{*}=m$, is another peculiarity of the Fayans method. Both features of this approach are closely related to the self-consistent Theory of Finite Fermi Systems (TFFS) [18]. The latter is based on general principles of the TFFS [19] supplemented with the TFFS selfconsistency relations [20]. These two peculiarities of the Fayans approach reflect, in an effective manner, energy dependence effects inherent to the self-consistent TFFS. E.g., the effective mass in this approach is a product $m^{*}=m_{k}^{*} \cdot m_{E}^{*}$ of the " $k$-mass" and the " $E$-mass". The two effects compensate each other almost exactly [18] resulting in $m^{*} \simeq 1$. In the Skyrme HF method, the $E$-mass is identically equal to unit and the effective mass may deviate from unity.

Until recently, the Fayans method was applied for spherical nuclei only. It turned out to be rather successful in systematic description of nuclear magnetic [21, 22] and quadrupole $[23,24]$ moments, nuclear radii [25], betadecay probabilities [26], the energies and $B(E 2)$ values for the first excited $2^{+}$states in semi-magic nuclei $[27,28]$. In a recent study of the single-particle energies in seven magic nuclei, a record accuracy was achieved [29]. Most of these calculations were made with the EDF DF3-a [30], which is a small modification of the DF3 $[15,17]$ param- 
eter set, concerning the spin-dependent terms of the EDF. In particular, the effective tensor term of DF3-a is significantly stronger than the DF3 one.

The Fayans EDF was applied recently to deformed nuclei for the first time [31]. A localized version $\mathrm{FaNDF}^{0}[16]$ of the general finite range Fayans EDF was used which makes its surface term more similar to the Skyrme one. This allowed to employ the computer code HFBTHO [32], developed for Skyrme EDFs, with some modifications.

The results obtained with the $\mathrm{FaNDF}^{0} \mathrm{EDF}$ for $\mathrm{Pb}$ and $U$ isotopic chains turned out to be promising. In the present work, we continue these calculations around the uranium region. In particular, we investigate the twoneutron drip line for a set of elements from $\mathrm{Pb}$ up to $\mathrm{Fm}$, those with even proton number being considered. For a systematic comparison, we carried out also all calculations for two popular Skyrme EDFs, SkM* [6] and SLy4 [7]. For completeness, we included also into the analysis predictions from the HFB-17 and HFB-27 functionals taken from Ref. [10]. In addition we discuss deformation characteristics of the drip line nuclei.

Recent interest to the problem of fixing the neutron drip line $[33,34]$ is partially induced with importance of this characteristic of the nuclear chart for analysis of the $r$-process dynamics in stars [35-37]. A couple of remarks should be made concerning validity of the EDF method with fixed set of parameters for predicting the drip lines. In particularly for the older generation EDFs, the parameterizations were obtained by using data on nuclei close to the stability, that is, with the chemical potential $\mu_{n, p} \simeq-8 \mathrm{MeV}$. When extrapolating to very neutron rich systems, the role of EDF parameter errors becomes prominent $[33,38]$. Also, analysis of the problem on the basis of the many-body theory point of view $[39,40]$ shows that in vicinity of drip lines, that is $\mu_{n} \rightarrow 0$ or $\mu_{p} \rightarrow 0$, the EDF parameters describing the effective $N N$-interaction at the nuclear surface may need to be modified. Close to the neutron drip line, with a small chemical potential $\mu_{n}$, attractive part of the $N N$-interaction may become enhanced, resulting a deeper neutron mean-field. This, as a conse-

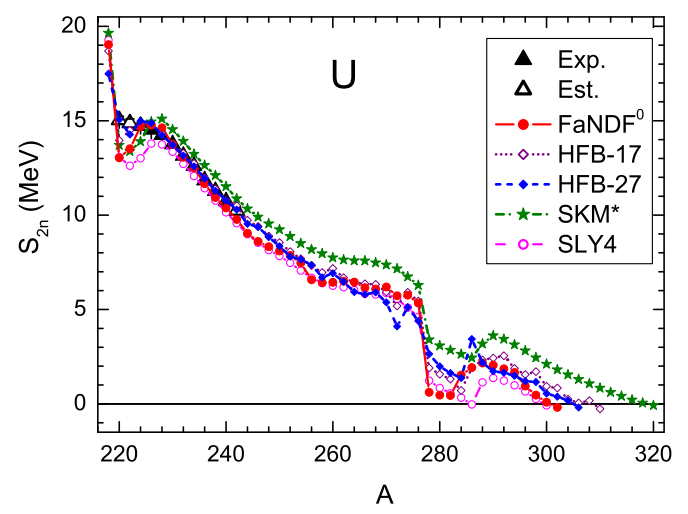

Figure 1. (Color online) $S_{2 n}$ values in the uranium chain for different EDFs. Experimental and estimated values are taken from Ref. [41].

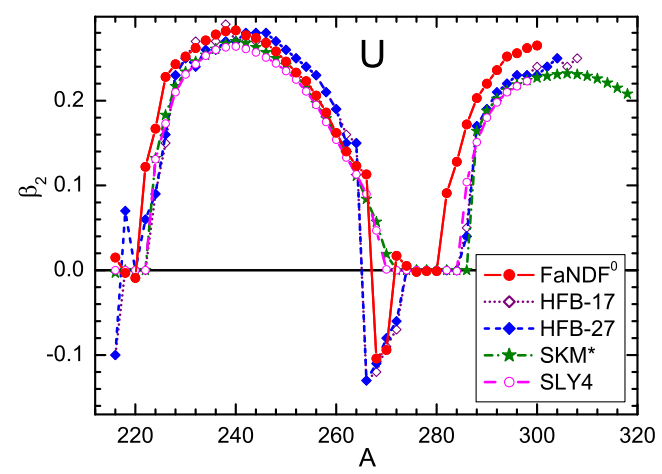

Figure 2. (Color online) $\beta_{2}$ values in the uranium chain for different EDFs.

quence, shifts the drip line farther away. To take this effect into account, a simple model was used in Refs. [39, 40] which, evidently, overestimates the effect, predicting unrealistically strong shift of the drip line. It was, nevertheless, demonstrated that this effect shifts the drip line. However, one should bear in mind that by explicitly introducing such kind of component to the EDF model, the model parameters need to be readjusted, which, so far, has not yet been done. Thus, the total net effect remains still unknown. In the present work we use the standard approach with fixed EDF parameters.

\section{Deformation properties of $\mathrm{Th}, \mathrm{U}$, and $\mathrm{Pu}$ isotopic chains}

Details of the computation scheme are identical to those described in Ref. [31]. We employ the axial computer code with the oscillator basis, the number of the oscillator shells being equal to $N_{\mathrm{sh}}=25$. We limit ourselves to the quadrupole deformation $\beta_{2}$ only, with reflection symmetry assumed. All the parameters of the normal component of the used $\mathrm{FaNDF}^{0} \mathrm{EDF}$ are the same as in Ref. [16]. As for the anomalous term of the EDF [16],

$$
\mathcal{E}_{\mathrm{anom}}=\sum_{i=n, p} v_{i}^{\dagger}(\mathbf{r}) F^{\xi}\left(\rho_{+}(\mathbf{r})\right) v_{i}(\mathbf{r})
$$

where $v_{i}(\mathbf{r})$ is the anomalous density, a simplified version is used,

$$
F^{\xi}\left(\rho_{+}\right)=C_{0}\left(f_{\mathrm{ex}}^{\xi}+h^{\xi}\left(\rho_{+} / \rho_{0}\right)\right) .
$$

Here $\rho_{+}=\rho_{n}+\rho_{p}$, and $C_{0}=\pi^{2} / p_{\mathrm{F}} m$ is the usual for TFFS normalization factor. The HFB equations are solved by using quasi-particle cut-off energy $E_{\text {cut }}=60 \mathrm{MeV}$. The main part of calculations are carried out by employing simplest ("volume") model of pairing, $h^{\xi}=0$, with $f_{\mathrm{ex}}^{\xi}=-0.440$. All calculations are repeated for two Skyrme EDFs, SkM* and SLy4, the results being compared also with predictions [10] from another two Skyrme EDFs, HFB-17 and HFB-27.

Let us begin from the uranium chain. The two-neutron separation energies,

$$
S_{2 n}(N, Z)=B(N, Z)-B(N-2, Z),
$$




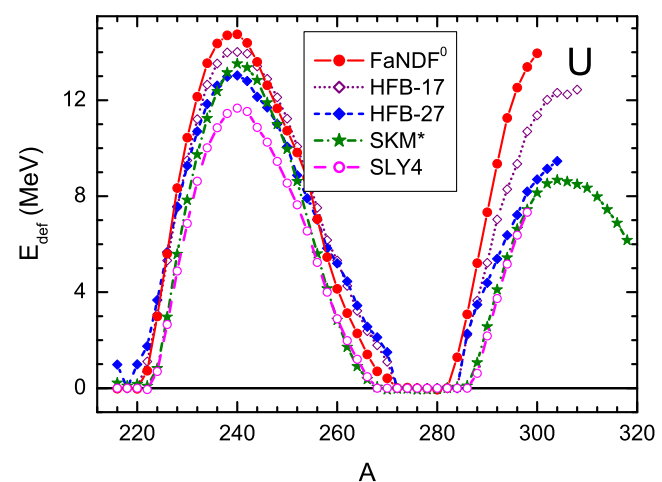

Figure 3. (Color online) $E_{\text {def }}$ values in the uranium chain for different EDFs.

are displayed in Fig. 1. Here $B(N, Z)$ is the binding energy of the nucleus under consideration. Comparison is made with experimental data [41] and predictions from four Skyrme EDFs. We first consider region of $A \leqslant 244$ with known experimental values. The HFB-17 and HFB27 models reaffirm their high accuracy. As to the FaNDF ${ }^{0}$ functional, agreement also looks rather reasonable, taking into account that the parameters were fitted [16] only for spherical nuclei not heavier than lead. The deviation of $0.5 \mathrm{MeV}$ from the experimental $S_{2 n}$ values for heavy $\mathrm{U}$ isotopes is explained mainly by two reasons, with is the use of a simple volume pairing interaction, and absence of the effective tensor term in the FaNDF ${ }^{0}$ EDF. Indeed, as it was shown in Ref. [30], the tensor term is especially important in uranium and transuranium region as, in corresponding spherical case, high- $j$ levels dominate in vicinity of the Fermi level for these nuclei. As a result, the spin-orbit density, which comes to the EDF together with the tensor force, is typically large in these nuclei, changing significantly along the isotopic chain. For the SLy4 EDF agreement is a bit worse, whereas the disagreement is more significant in the $\mathrm{SkM}^{*}$ case. It is worth to note that this Skyrme EDF, being fitted to masses not with so high accuracy as some modern equivalents, reproduces e.g. fission properties in actinides or energies and $B(E 2)$ values of the first excited $2^{+}$states in semi-magic nuclei [42] relatively well. For higher $A$ values the $\mathrm{SkM}^{*}$ values of $S_{2 n}$ are significantly higher than those for all other EDFs. As a result the corresponding drip line point $A_{2 n}^{\text {drip }} \simeq 320$ turns out be significantly farther away than for all other EDFs. This quantity is defined as the last nucleus for which the two-neutron separation energy is yet positive. For SLy4 we note that $S_{2 n}$ at $A=286$ is slightly negative. However, after this point the $S_{2 n}$ remain positive up to $A=298$. This kind binding re-entrance was also predicted at [33]. Thus, we can set $A_{2 n}^{\text {drip }}$ (SLy4) $=298$.

The ground state quadrupole deformation parameter $\beta_{2}$ of the $\mathrm{U}$ chain and the corresponding deformation energy,

$$
E_{\text {def }}\left(\beta_{2}\right)=B\left(\beta_{2}\right)-B\left(\beta_{2}=0\right),
$$

are displayed in Figs. 2 and 3, correspondingly. Here, each curve is cut around the corresponding drip point. Generally, all five EDFs under discussion behave in similar way,

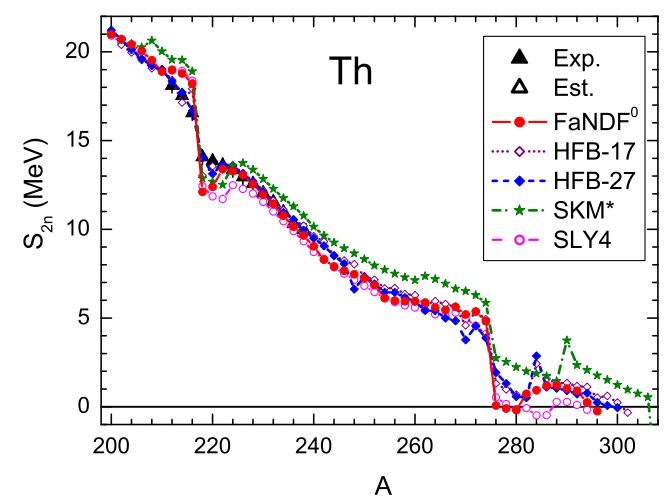

Figure 4. (Color online) $S_{2 n}$ values in the thorium chain for different EDFs.

the most of nuclei in this chain being deformed in a prolate way whereas there exists a region of spherical nuclei around $A \simeq 280$. In more detail, the width of the spherical region is narrowest for the $\mathrm{FaNDF}^{0} \mathrm{EDF}$ and widest for SLy4. Both of the HFB EDFs predict oblate deformations for four nuclei $262 \leqslant A \leqslant 268$, whereas the $\mathrm{FaNDF}^{0} \mathrm{EDF}$, only for two of them, $A=264$ and $A=266$.

The reason for this difference is quite simple. In vicinity of the phase transition, with a change of the deformation sign, there are typically two energetically close by minima, the prolate and oblate one. Their closeness may be confirmed with the observation that the deformation energy, see Fig. 3, does not show any non-regular behavior in the transition point, where the order of two minima changes. In such kind of situation, the transition value of $A$ may move even due to a small change of the EDF parameters. In the mass region of $A>280$ the prolate deformation arises for the $\mathrm{FaNDF}^{0} \mathrm{EDF}$ for three points earlier than for both HFB and SLy4 EDFs. In this region the maximum value of $\beta_{2} \simeq 0.25$ appears for FaNDF ${ }^{0}$ EDF just in the drip line point. The corresponding value is a bit less for the HFB-17 EDF, being $\beta_{2} \simeq 0.2$ for other three EDFs. The difference is greater for the deformation energy due to its quadratic behavior around the minima. Thus, all five EDFs under consideration predict a well developed prolate deformation for uranium isotopes in the vicinity of the drip line.

Next, we investigate the thorium chain. The twoneutron separation energies are displayed in Fig. 4. Again, all EDFs, with exception of SkM*, behave in general similarly in the region of $A \leqslant 280$, the latter curve being significantly higher. Close to the drip line, however, the picture is different. In accordance with the above discussion, binding re-entrance can be now seen also for FaNDF ${ }^{0}$. As a result, we obtain $A_{2 n}^{\text {drip }}\left(\mathrm{FaNDF}^{0}\right)=294$. For the HFB-17 and HFB-27 EDFs, the drip points are $\simeq 300$ whereas again the highest value $A_{\text {drip }}=306$ there is for the $\mathrm{SkM}^{*} \mathrm{EDF}$.

The ground state deformation parameter $\beta_{2}$ of the Th chain and the corresponding deformation energy are displayed in Figs. 5 and 6, correspondingly. Again all five EDFs lead to similar results until $A \simeq 280$, and there is rather extended region of spherical nuclei around this 


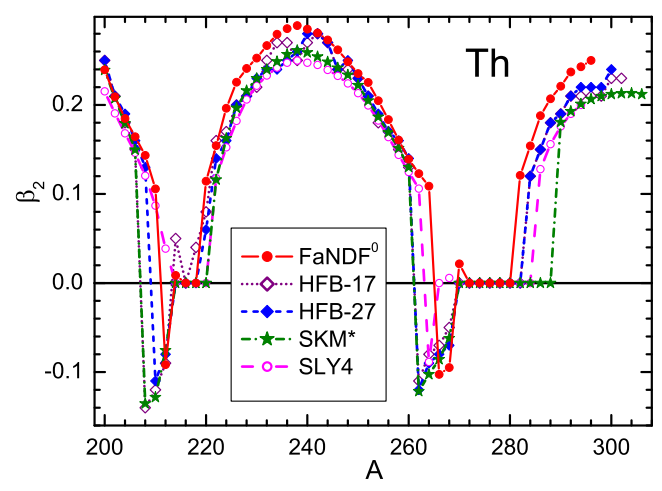

Figure 5. (Color online) $\beta_{2}$ values in the thorium chain for different EDFs.

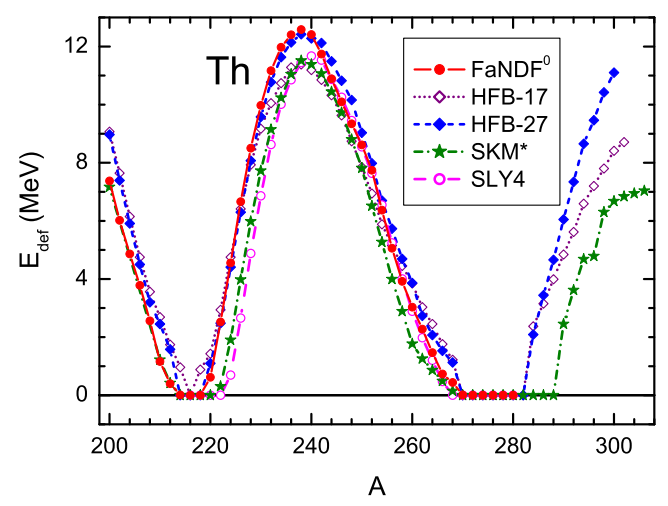

Figure 6. (Color online) $E_{\text {def }}$ values in the thorium chain for different EDFs.

point. For all EDFs under consideration, the positive deformations appear in the drip region, the values of deformation parameter reaching $\beta_{2} \simeq 0.2$.

Lastly, we investigate the plutonium isotopic chain. The two-neutron separation energies are shown in Fig. 7. Qualitatively, the picture reminds very much that for the $\mathrm{U}$ case, see Fig. 1. Again, all EDFs except $\mathrm{SkM}^{*}$ reproduce reasonably the experimental data, and for the $\mathrm{SkM}^{*}$, the two-neutron separation energies are higher, especially in the drip region. Again the corresponding drip line point value $A_{\text {drip }}\left(\mathrm{SkM}^{*}\right)=324$ is much higher that those for other EDFs.

The deformation parameter $\beta_{2}$ and the corresponding deformation energy $E_{\text {def }}\left(\beta_{2}\right)$ are shown in Figs. 8 and 9, correspondingly. The HFB-17 and HFB-27 EDF reveal a region of oblate deformations at $266 \leqslant A \leqslant 268$. None of other EDFs confirm it. Again, this disagreement is a consequence of a competition of two close by energy minima, as was discussed earlier. For HFB-17 and HFB-27 EDFs, the oblate minimum has lower energy, whereas for other EDFs, the prolate minimum has a lower energy. Similarly as in the uranium chain, the spherical region ends for the $\mathrm{FaNDF}^{0} \mathrm{EDF}$ a bit earlier than for others. One more peculiarity occurs for the Fayans EDF: after the last spherical nucleus ${ }^{280} \mathrm{Pu}$, a small oblate deformation $\beta_{2} \simeq-0.05$ appears in ${ }^{282} \mathrm{Pu}$, which than becomes prolate deformed, $\beta_{2} \simeq 0.1 \mathrm{in}{ }^{284} \mathrm{Pu}$. In this case, there is a competition of three

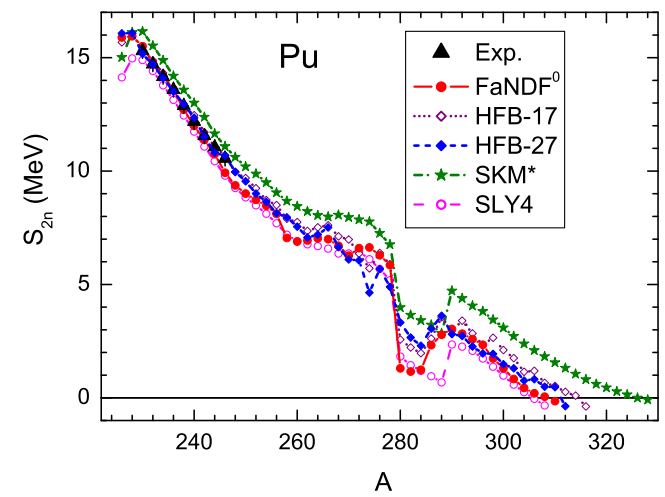

Figure 7. (Color online) $S_{2 n}$ values in the plutonium chain for different EDFs.

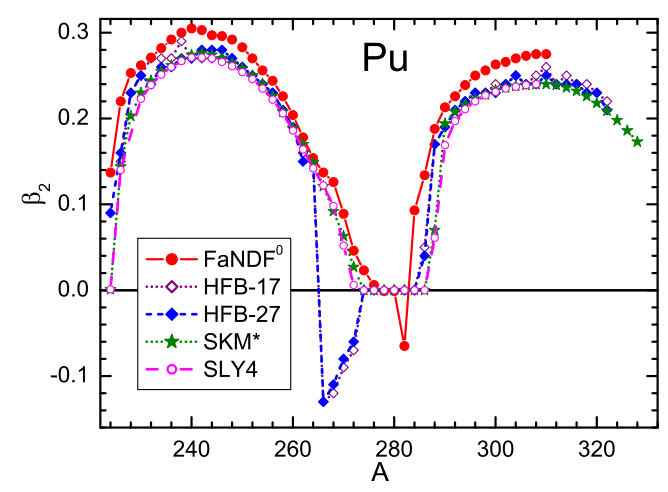

Figure 8. (Color online) $\beta_{2}$ values in the plutonium chain for different EDFs.

close by minima, a spherical one and two deformed ones. The spherical minimum is lowest in ${ }^{280} \mathrm{Pu}$, the oblate one in ${ }^{282} \mathrm{Pu}$ and the prolate minimum in ${ }^{284} \mathrm{Pu}$. Note that such a small negative deformation in ${ }^{282} \mathrm{Pu}$ is almost invisible in the deformation energy curve, in Fig. 9. Similarly as in the uranium chain, all the EDFs under consideration lead to a strong prolate deformations in the drip line region, and again the FaNDF ${ }^{0}$ deformation is the largest one.

\section{Two-neutron drip line}

In this section, we analyze the two-neutron drip line for a set of elements with an even $Z$ value, from $\mathrm{Pb}$ up to Fm. These nuclei are important for analysis of the $r$-process in stars [35-37]. As in the previous Sec. 2, we compare predictions from the $\mathrm{FaNDF}^{0}$ functional with those from four Skyrme EDFs. The results are presented in Table 1. The values in the SLy4 and SkM* columns are found using the code [32]. They coincide or are very close to the corresponding results presented in Ref. [33]. Small differences can be explained with some distinctions in the calculation details, for example by the number of used oscillator shells. The values in HFB-17 and HFB-27 are taken from [10]. For each $A_{2 n}^{\text {drip }}$, the corresponding value of the deformation parameter is given in brackets. We see that the major part of the EDFs under consideration predict a spherical form for the drip line nuclei in the region from 
Table 1. Two-neutron drip line points $A_{2 n}^{\text {drip }}(Z)$, from $\mathrm{Pb}$ to Fm, for different EDFs. In addition, the deformation $\beta_{2}$ of the drip line nucleus is given in brackets.

\begin{tabular}{cccccc}
\hline Element & FaNDF $^{0}$ & SLy4 & SkM $^{*}$ & HFB-17 & HFB-27 \\
\hline $\mathrm{Pb}$ & $266[0.00]$ & $266[0.00]$ & $272[0.00]$ & $266[0.00]$ & $266[0.00]$ \\
$\mathrm{Po}$ & $270[0.00]$ & $272[0.00]$ & $280[0.00]$ & $268[0.00]$ & $268[0.00]$ \\
$\mathrm{Rn}$ & $268[0.00]$ & $274[0.00]$ & $298[0.20]$ & $272[0.00]$ & $274[0.00]$ \\
$\mathrm{Ra}$ & $272[0.00]$ & $276[0.00]$ & $304[0.21]$ & $>286$ & $>286$ \\
$\mathrm{Th}$ & $294[0.24]$ & $292[0.19]$ & $306[0.21]$ & $300[0.23]$ & $298[0.22]$ \\
$\mathrm{U}$ & $300[0.26]$ & $298[0.22]$ & $318[0.21]$ & $308[0.25]$ & $304[0.24]$ \\
$\mathrm{Pu}$ & $308[0.27]$ & $304[0.24]$ & $324[0.20]$ & $314[0.25]$ & $310[0.25]$ \\
$\mathrm{Cm}$ & $312[0.28]$ & $310[0.25]$ & $336[0.14]$ & $320[0.24]$ & $316[0.24]$ \\
$\mathrm{Cf}$ & $318[0.27]$ & $316[0.25]$ & $354[0.00]$ & $324[0.24]$ & $322[0.24]$ \\
$\mathrm{Fm}$ & $324[0.26]$ & $322[0.24]$ & $342[0.23]$ & $330[0.23]$ & $328[0.23]$ \\
\hline
\end{tabular}

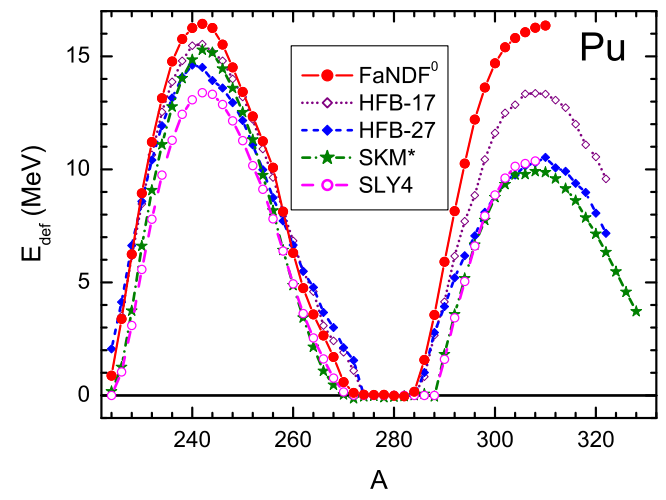

Figure 9. (Color online) $E_{\mathrm{def}}$ values in the plutonium chain for different EDFs.

$\mathrm{Pb}$ until $\mathrm{Ra}$, whereas all of them, from $\mathrm{Th}$ and heavier, are deformed.

As a rule, predictions from the $\mathrm{SkM}^{*} \mathrm{EDF}$ are significantly higher compared to others. The difference is especially large for $\mathrm{Rn}$ and $\mathrm{Ra}$ elements for which this EDF predicts a strong deformation in the drip line region, whereas these nuclei remain to be spherical for other EDFs. For the SkM* EDF, a strong irregularity in the $A_{2 n}^{\text {drip }}(Z)$ dependence occurs for Cf. The anomalously high value of $A_{2 n}^{\text {drip }}\left(\mathrm{SkM}^{*}\right)$ is explained with the the competition between the spherical and prolate minima of the total energy, the spherical one being lower, whereas the prolate ones are lower in all the neighboring elements. The predictions from the HFB-17 and HFB-27 EDFs are, as a rule, rather close to each other. The maximal difference between the corresponding values of $A_{2 n}^{\text {drip }}$ equals to 4 . As to the $\mathrm{FaNDF}^{0}$ predictions, they turned out to be very close to those of the SLy4 EDF. The difference between them and both HFB EDFs is also quite moderate.

A remark should be made concerning the relation between $A_{2 n}^{\text {drip }}$ values and one-neutron drip line points $A_{n}^{\text {drip }}$. Usually, due to the pairing effect, the inequality $A_{n}^{\text {drip }} \leqslant A_{2 n}^{\text {drip }}$ is valid [33, 34].

Finally, we would like to stress, that in particularly such a heavy region of the nuclear chart, extrapolation of current EDF models up to the drip line is prone to large uncertainties. Therefore, the results presented here should be taken with a typical uncertainty bar of \pm 10 mass units, or more [33]. An additional shift of the two-neutron drip line may occur due to the effect of $\mu$-dependence of the EDF parameters $[39,40]$, as discussed in the Introduction.

\section{Conclusions}

Fayans energy density functional $\mathrm{FaNDF}^{0}$ is applied to nuclei around the uranium region. For $\mathrm{Th}, \mathrm{U}$, and $\mathrm{Pu}$ isotopic chains, the two-neutron separation energies $S_{2 n}$, the ground state quadrupole deformation parameter $\beta_{2}$ and the corresponding deformation energies $E_{\text {def }}\left(\beta_{2}\right)$ are found and compared with predictions from several Skyrme EDFs. Those from the SLy4 and SkM* EDFs were calculated by using the code [32], whereas the HFB-17 and HFB-27 predictions are taken from [10]. For the major part of nuclei with known experimental $S_{2 n}$ values, the results obtained with the Fayans and SLy4 EDFs are rather close to those with two HFB EDFs, the HFB-17 one being highly accurate in the overall description of nuclear masses [9]. The SkM* EDF overestimates $S_{2 n}$ values leading for these three elements to the two-neutron drip line point $A_{2 n}^{\text {drip }}$ values noticeably higher than for those obtained with the other EDFs under consideration. The deformation characteristics, the deformation parameter $\beta_{2}$ and the corresponding deformation energy $E_{\text {def }}\left(\beta_{2}\right)$, for the $\operatorname{FaNDF}^{0}$ $\mathrm{EDF}$, are also in the overall agreement with those of SLy4 and two the HFB EDFs. The $\mathrm{SkM}^{*}$ predictions are again different, especially around the drip line region.

The two-neutron drip line is found for $\mathrm{FaNDF}^{0}, \mathrm{SLy} 4$ and $\mathrm{SkM}^{*}$ EDFs for a set of elements with even proton number, from $\mathrm{Pb}$ up to $\mathrm{Fm}$. This part of the nuclear chart is important for the study of the $r$-process in stars [3537]. The consideration is made within a standard approach with fixed EDF parameters found mainly for stable nuclei. Within such approach, there is an overall agreement between all EDFs under consideration except $\mathrm{SkM}^{*}$, the latter predicting sufficiently higher $A_{2 n}^{\text {drip }}$ values.

To conclude, the ground state properties of deformed nuclei in the uranium region, predicted from the $\mathrm{FaNDF}^{0}$ EDF, are found to be rather similar to those from several popular Skyrme EDFs. The same also holds for the predic- 
tion of the two-neutron drip line for even proton number elements from $\mathrm{Pb}$ to $\mathrm{Fm}$. With such estimates, however, one should be careful since they contain a lot of uncertainties. Nevertheless, the $\mathrm{FaNDF}^{0}$ prediction for the drip line seems to be in the line with typical Skyrme EDFs.

\section{Acknowledgements}

The work was partly supported by the Grant NSh-932.2014.2 of the Russian Ministry for Science and Education, by the RFBR Grants No. 13-02-00085-a, No. 13-02-12106_ofi-m, No. 14-0200107-a, No. 14-22-03040_ofi-m, the Grant by IN2P3-RFBR under Agreement No. 11-02-91054, and Swiss National Scientific Foundation Grant No. IZ73Z0_152485 SCOPES. This work was also supported (M.K.) by Academy of Finland under the Centre of Excellence Programme 2012-2017 (Nuclear and Accelerator Based Physics Programme at JYFL) and FIDIPRO program. Calculations are partially made on the Computer Center of NRC "KI".

\section{References}

[1] D. Vautherin and D. M. Brink, Phys. Rev. C 5, 626 (1972)

[2] J. Dechargé and D. Gogny, Phys. Rev. C 21, 1568 (1980)

[3] P. Ring, Prog. Part. Nucl. Phys. 37, 193 (1996)

[4] W. Kohn and L. J. Sham, Phys. Rev. 140, A1133 (1965)

[5] P. Hohenberg and W. Kohn, Phys. Rev. 136, B864 (1964)

[6] J. Bartel, P. Quentin, M. Brack, C. Guet, H.-B. Håkansson, Nucl. Phys. A 386, 79 (1982)

[7] E. Chabanat et al., Nucl. Phys. A 635, 231 (1998)

[8] M. Bender, P.-H. Heenen, P.-G. Reinhard, Rev. Mod. Phys. 75, 121 (2003)

[9] S. Goriely, N. Chamel, J. M. Pearson, Phys. Rev. Lett. 102, 152503 (2009)

[10] S. Goriely, [http://www-astro.ulb.ac.be/bruslib].

[11] M. Kortelainen et al., Phys. Rev. C 85, 024304 (2012)

[12] M. Baldo, P. Schuck, X. Viñas, Phys. Lett. B 663, 390 (2008)

[13] M. Baldo, L. M. Robledo, P. Schuck, X. Viñas, Phys. Rev. C 87, 064305 (2013)

[14] A. V. Smirnov, S. V. Tolokonnikov, S. A. Fayans, Sov. J. Nucl. Phys. 48, 995 (1988)

[15] I. N. Borzov, S. A. Fayans, E. Kromer, D. Zawischa, Z. Phys. A 355, 117 (1996)

[16] S. A. Fayans, JETP Lett. 68, 169 (1998)

[17] S. A. Fayans, S. V. Tolokonnikov, E. L. Trykov, D. Zawischa, Nucl. Phys. A 676, 49 (2000)
[18] V. A. Khodel and E. E. Saperstein, Phys. Rep. 92, 183 (1982)

[19] A. B. Migdal, Theory of Finite Fermi Systems and Applications to Atomic Nuclei (Wiley, New York, 1967)

[20] S. A. Fayans and V. A. Khodel, JETP Lett. 17, 444 (1973)

[21] I. N. Borzov, E. E. Saperstein, S. V. Tolokonnikov, Phys. At. Nucl. 71, 469 (2008)

[22] I. N. Borzov, E. E. Saperstein, S. V. Tolokonnikov, G. Neyens, N. Severijns, Eur. Phys. J. A 45, 159 (2010)

[23] S. V. Tolokonnikov, S. Kamerdzhiev, S. Krewald, E. E. Saperstein, D. Voitenkov, Eur. Phys. J. A 48, 70 (2012)

[24] S. Kamerdzhiev, S. Krewald, S. Tolokonnikov, E. E. Saperstein, D. Voitenkov, Eur. Phys. J. Web Conf. 38, 10002 (2012)

[25] E. E. Saperstein and S. V. Tolokonnikov, Phys. At. Nucl. 74, 1277 (2011)

[26] I. N. Borzov, Phys. Rev. C 67, 025802 (2003); Phys. Rev. C 71, 065801 (2005)

[27] S. V. Tolokonnikov, S. Kamerdzhiev, D. Voytenkov, S. Krewald, E. E. Saperstein, Phys. Rev. C 84, 064324 (2011)

[28] S. V. Tolokonnikov, S. Kamerdzhiev, S. Krewald, E. E. Saperstein, D. Voitenkov, Eur. Phys. J. Web Conf. 38, 04002 (2012)

[29] N. V. Gnezdilov, I. N. Borzov, E. E. Saperstein, S. V. Tolokonnikov, Phys. Rev. C 89, 034304 (2014)

[30] S. V. Tolokonnikov and E. E. Saperstein, Phys. At. Nucl. 73, 1684 (2010)

[31] S. V. Tolokonnikov, I. N. Borzov, M. Kortelainen, Yu. S. Lutostansky, E. E. Saperstein, J. Phys. G: Nucl. Part. Phys. 42, 075102 (2015)

[32] M. V. Stoitsov, N. Schunck, M. Kortelainen et al., Comp. Phys. Commun. 184, 1592 (2013)

[33] J. Erler, N. Birge, M. Kortelainen et al., Nature 486, 509 (2012)

[34] A. V. Afanasjev, S. E. Agbemava, D. Ray, P. Ring, Phys. Rev. C 91, 014324 (2015)

[35] T. Kajino, S. Wanajo, G. J. Mathews, Nucl. Phys. A 704, 165 (2002)

[36] I. V. Panov, Astron. Lett. 29, 163 (2003)

[37] I. Petermann et al., Eur. Phys. J. A 48, 122 (2012)

[38] M. Kortelainen, J. Phys. G: Nucl. Part. Phys. 42, 034021 (2015)

[39] M. Baldo, U. Lombardo, E. E. Saperstein, M. V. Zverev, Phys. Lett. B 533, 17 (2002)

[40] E. E. Saperstein and S. V. Tolokonnikov, JETP Lett 78, 343 (2003)

[41] G. Audi, A. H. Wapstra, C. Thibault, Nucl. Phys. A 729, 337 (2003)

[42] J. Terasaki, J. Engel, G. F. Bertsch, Phys. Rev. C 78, 044311 (2008) 